\title{
environnement et pose d'une conduite sous-marine
}

\author{
par \\ A. Grovel \\ I.U.T. Génie civil, Saint-Nazaire
}

RESUME - La pose d'une conduite sous-marine de 2,3 km de long et 250 mm de diamètre, près de Lorient, dans une région particulièrement exposée à la houle, fut guidée par l'étude de l'environnement, l'océanographie fixa la zone d'arrivée en mer, l'analyse des houles et l'environnement humain imposèrent le point de dénart, et c'est l'utilisation d'une caractéristique géologique du site qui permit le franchissement en sécurité du déferlement. Des mesures postérieures ont montré que le déferlement pouvait s'accompagner à cet endroit de phénomènes annexes plus dangereux que ceux initialement prévus ; on peut donc penser que c'est la disposition particulière du passage sous les déferlantes qui assure denuis bientôt dix ans la bonne tenue de l'ouvrage.

\section{INTRODUCTION}

En -1967 la ville de LORIENT envisageait de résoudre définitivement les problèmes de pollution de sa rade, dans laquelle se jetaient tous les égouts, en construisant une station d'épuration dont les rejets, stérilisés à $100 \%$, seraient évacués au large, sur le fond, en un point de non retour. M. G.SAURY et M. M.RUZ, qui étaient alors respectivement Ingénieur d'Arrondissement et Ingénieur T.P.E. responsable des V.R.D. de la ville, chargèrent alors l'auteur de cette communication de l'étude préliminaire en nature de cet émissaire sous marin. Il s'agissait de définir les contraintes de l'environnement sur les choix du traçé, de la mise en oeuvre et de la conception de 1 'ouvrage.

Une convention entre I'Arrondissement Maritime de l'Equipement du Morbihan et le laboratoire de Géologie Marine de l'Université de Nantes (Directeur F. OTTMANN) a permis d'utiliser tous les moyens d'investigation nécessaires.

\section{LE SITE}

La carte ci-après montre la situation du projet et les faits marquants à priori. A l'intérieur des terres, dans la rade de Lorient, se jettent les estuaires du BLAVET et du SCORFF, dont les crues quasi simultanées sont susceptibles d'augmenter considérablement la compétence d'un courant de jusant.

La géologie est simple, tout au moins pour ses utilisations en travaux nublics. La bande côtière est formée de granulite, ou granite à deux micas, diaclasé et souvent altéré ; à quelques kilomètres de là, à PLOEMEUR, se situent les plus grandes carrières françaises de kaolin. Le recouvrement superficiel est généralement absent, hormis quelques lambeaux de conglomérats pliocènes en falaise sur les bords du BLAVET et à la sortie même de la rade de LORIENT. On cherche vainement sur cette côte Sud de la Bretagne les débris de coulées de solifluxion, et notamment les blocs erratiques, vestiges des glaciations quaternaires, si fréquents sur la côte Nord. Au large, I'île de GROIX, aux célébres micashistes et amphibolites, assure une protection contre les houles du Sud Ouest; une ligne de haut fonds la relie aux îles GLENANS, et cette ligne est interrombue par des passages ouverts aux houles de ouest sud ouest.

Entre GROIX et la côte, les fonds sousmarins peuvent être schématisés comme un plateau de profondeur 25 mètres, entaillé à l'Ouest par une fosse ouverte vers le large par un de ces passages et profonde d'environ 40 mètres, la fosse du TALUD. A 2300 mètres, dans l'Est Nord Est, le point le plus proche est la pointe du TALUD; la liaison entre la pointe et la fosse du même nom parât à priori la plus intéressante pour un émissaire, avec le maximum de trajet terrestre et le minimum sous-marin. 
Le premier point de l'étude a donc consisté à vérifier que la fosse du TALUD Douvait être considérée comme un point de nonretour, par des mesures portant sur la stratification des eaux, le régime des courants et la sédimentologie. Ce point une fois acquis, restait à définir l'ouvrage.

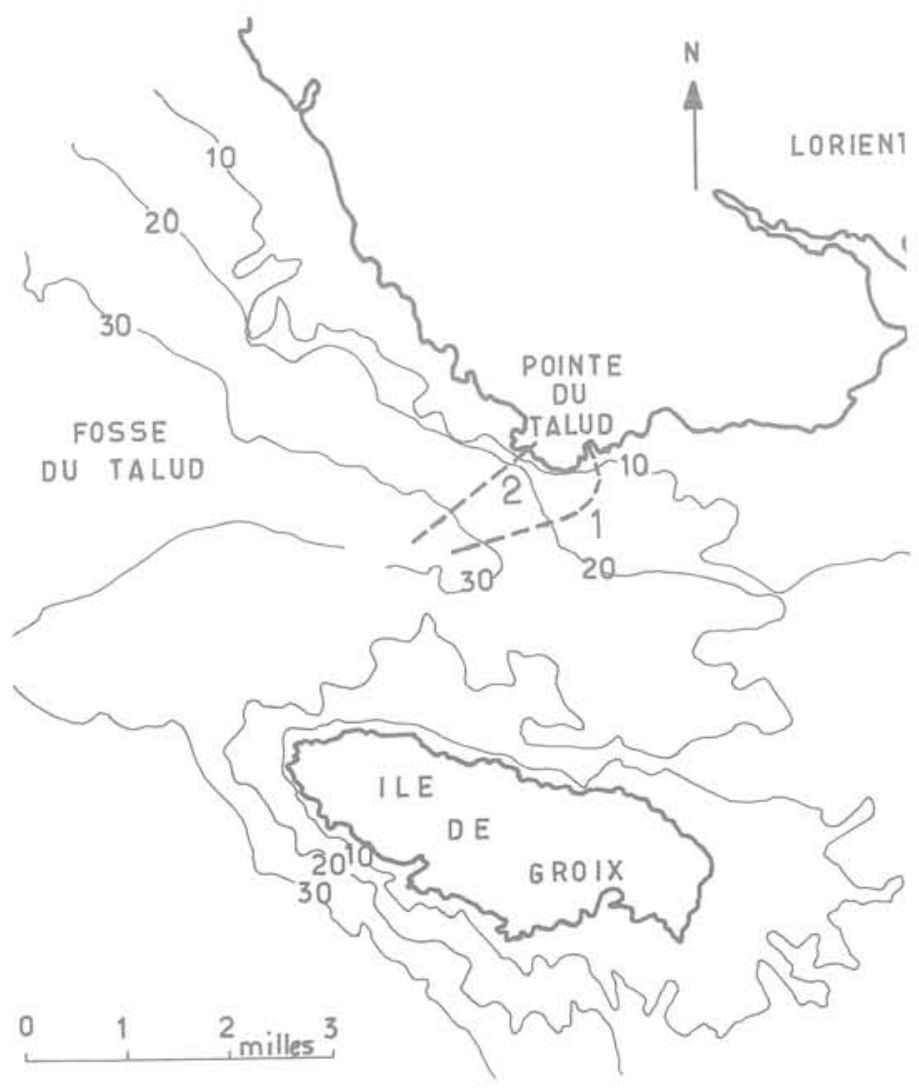

LES FORCES PHYSIQUES

\section{Les courants}

Les mesures anciennes demandaient à être complétées. On réalise donc dans ces "coureaux" un mouillage de courantographe de 16 jours, trois stations au point fixe de 25 heures, trois coupes verticales et deux lâchers de 10 flotteurs en surface. La corrélation avec la météorologie permit d'analyser convenablement les courants entre leurs différentes composantes :

- Mi-jusant d'une marée de coefficient120

- Courant de dérive du plus fort vent d'Est recensé en 10 ans.
- Crues centenaires du BLAVET et du SCORFF - Courant de pente calculé et extranolé d'après 10 ans d'observations météorologiques.

On atteignait des vitesses maximales de 2 noeuds, soit 1 mètre/seconde, portant à

l'ouest, sur les fonds destinés à porter la conduite.

La nécessité de l'ancrage par pieux n'annaraissait donc nullement et la relative modération des courants autorisait une solution où l'alourdissement de l'émissaire suffirait à le stabiliser.

\section{Les houles}

Deux mois de mesures par fonds de 25 mètres à l'aide d'un canteur à pression nermirent de poser le problème et notamment de commencer une corrélation houle-météorologie, en utilisant la méthode de calcul SVERDRUP MUNK-BRETSCHNEIDER sur 10 ans de cartes météorologiques de l'Atlantiaue. Les plans de houles nour les directions princinales et les périodes les plus fréquentes mirent en lumière 1'importance des houles venant de l'Ouest sud Ouest ; cette direction coincidait de plus aver celle des vents locaux les plus fréquents et les plus forts. Il y avait donc, lors des tempêtes, superposition de la houle et de la "mer du vent", avec le phénomène habituel de concentration autour d'une pointe comme celle du TALUD.

La houle de projet était donc la suivante : Période : 10 - 11 secondes Direction : Ouest-Sud-Ouest

Occurence de hauteur : 5 mètres annuelle 10 mètres décennale Après réfraction, sur zone, par fonds de 25 mètres.

Vu l'importance des houles il fallait impérativement présenter la conduite orthogonalement aux crêtes des houles les nlus fortes ; l'orientation vers I'Ouest-Sudouest était donc imposée. 


\section{CHOIX DU TRACE}

Compte tenu des conditions précédentes, auxquelles l'ouvrage devra résister, c'est l'étude des fonds marins tant du point de vue morphologique que géologique et géotechnique qui décidera du choix définitif du traçé.

En effet l'importance des houles et surtout la nécessité de franchir le déferlement, zone toujours critique, ont fait immédiatement songer à la solution d'une conduite ensouillée.

Les fonds marins entre GROIX et la côte étaient très bien décrits par des minutes de sonde, au $1 / 5000$, exécutées au plomb suiffé, au siècle dernier, par le Service Hydrographique. Un relevé bathymétrique, avec des moyens modernes, permit de se recaler sur ces documents. Des prélèvements (une cinquantaine) à la draque, à la benne, au carottier KULLENBERG, des plongées enfin, complétèrent ces informations.

Dans la solution de la canalisation ensoullée, le traçé ( $\mathrm{n}^{\circ} 1$ sur la carte) suivait des zones sableuses, en partant d'une crique particulièrement bien abritée des houles d'Ouest et d'Ouest sudOuest pour virer plus au large en se présentant dans le sens de la fosse du TALUD. L'argument favorable était la découverte sous la surface du sable, à une profondeur comprise entre un demimètre et un mètre, d'un niveau assez régulier de galets ; mais la mobilité probable de ce sable sous l'action de la houle faisait aussi prévoir qu'un pipeline simplement posé pourrait s'enfouir naturellement très doucement jusqu'aux galets.

De plus le trajet était long et surtout, la présence d'habitations à terre autour de la crique, interdisait la solution de pose la plus économique, à savoir l'implantation à terre du chantier d'assemblage de la conduite.

La solution adoptée ( $n^{\circ} 2$ sur la carte) consistera donc à s'orienter perpendiculairement aux crêtes des houles dominantes suivant le traçé le plus court, en partant de la pointe du TALUD, là où une diaclase dans la roche permettait d'atteindre le plus rapidement des fonds de 17 mètres. on présentait donc ainsi la plus petite longueur possible au déferlement. De plus, la pointe étant déserte, le chantier de préparation et de lancement était aisé à installer.
La pose d'une semelle filante telle qu'une canalisation de $250 \mathrm{~mm}$ de diamètre sur des fonds de roche, de sables recouvrant des galets, d'argiles raides altérations de granites sous-jacents, ne posaient aucun problème géotechnique sérieux.

Vis à vis des courants et des vitesses orbitales de la houle, le poids et les charges de la conduite enrobée de béton assuraient sa stabilité.

La partie fragile se situait évidemment dans la zone du déferlement. Or dans cette zone, par beau temps, le sondage moderne à l'ultra-son avait décrit un sol sousmarin de deux à trois mètres moins profond que ne le montraient les cartes du siècle dernier.

A terre, à deux cent mètres de là, sur les falaises de la nointe du TALUD, la surface de la roche granitique était plane, rabotée, avec des stries régulières, comme dans les pays nordiques actuels. S'agissait-il là de traces de coulées de solifluxion? Dans ce cas, qu'étaient-elles devenues ?

Une plongée permit de découvrir à l'endroit même où les écarts de sonde étaient si grands, au pied du flanc Ouest de la pointe, un chaos granitique composé de blocs de 5 tonnes : un "HUELGOAT" sous marin.

Sur les blocs le plomb des vieux sondeurs devait glisser, atteindre des profondeurs plus grandes que le sommet, alors que l'ultra son lissait le tout en un toit élevé. Pour franchir cet obstacle l'entreprise qui lança l'émissaire dut réaliser une saignée dans ce chaos en relevant ces blocs à la grue.

Or des mesures de houle récentes dans cette région (THOMAS-GROVEL) interprétées par le Laboratoire de Génie Civil de l'ENSM, ont montré que les"long-shore currents", ces courants pulsatoires très violents qui prennent naissance entre le déferlement et la côte, avait probablement, lors de l'étude de cette conduite, été très sous-estimés. En certains points, par tembêtes d'ouest, on pouvait craindre des vitesses de 6 noeuds, soit 3 mètres/seconde.

On peut donc s'interroger : $\mathrm{n}^{\text {'est ce pas }}$ la protection des blocs de granite du chaos dans la zone du déferlement et près de la côte, qui nermet à cet émissaire de si bien résister aux temnêtes en ce lieu très exposé, et cela depuis bientôt 10 ans ? 


\section{CONCLUSIONS}

L'étude de l'environnement a donc guidé pas à pas la recherche d'une solution de pose. Les vitesses de courants n'étaient pas excessives et permettaient d'envisager une conduite alourdie et protégée mais sans ancrage par pieux. Le sol sousmarin, lorsqu'il était mobilisable, n'était qu'un faible recouvrement de formations stables, constituant de bonnes fondations. Le gros problème était donc constitué par la houle. La direction de lancement fut celle de l'orthogonale des houles dominantes et le franchissement du déferlement fut assuré au point de pente maximale, en profitant au mieux de l'abri d'une tranchée au milieu d'un chaos de blocs de granite.

Les ruptures au déferlement étant très fréquentes dans ce genre d'ouvrages, on peut se demander si l'accumulation, de part et d'autre d'une conduite, d'enrochements naturels ou artificiels dans la zone des "long-shore currents", n'assurerait pas une solution de protection analogue.

\section{Références}

GROVEL A. (1970)

Etude d'un Estuaire dans son Environnement, Thèse Etat Fac. Sc. Nantes

THOMAS P. (1977)

Contribution à l'étude de la houle à l'embouchure de la Laİta

Rap. D.D.E. Morbihan

THOMAS P. , GROVEL A. (1978)

Problèmes posés par le dépouillement d'une houle irrégulière à la côte.

Communicat. Colloque U.O.F. 1978. 